\title{
Efficacy of Hataedock Treatments for Maintenance and Formation of Lipid Barrier in Obese NC/Nga Mice with Dermatophagoides Farinae-Induced Atopic Dermatitis
}

\author{
Hee-Yeon Kim ${ }^{1,4}$, Sang-Hyun $\mathrm{Ahn}^{2}$, In-Jun Yang ${ }^{3}$, Jin-Hong Cheon ${ }^{1,4}$, Kibong Kim ${ }^{1,4^{*}}$ \\ ${ }^{1}$ Department of Korean Pediatrics, School of Korean Medicine, Pusan National University, Pusandaehak-ro 49, Mulgeum-eup, \\ Yangsan-si, Gyeongsangnam-do 50612, Republic of Korea \\ ${ }^{2}$ Department of Anatomy, College of Korean Medicine, Semyung University, Semyung-ro 65, Jecheon-si, Chungcheongbuk-do \\ 27136, Republic of Korea \\ ${ }^{3}$ Department of Physiology, College of Korean Medicine, Dongguk University, Dongdae-ro 123, Gyeongju-si, Gyeongsangbuk-do \\ 38066, Republic of Korea \\ ${ }^{4}$ Department of Korean Pediatrics, Korean Medicine Hospital, Pusan National University, Geumo-ro 20, Mulgeum-eup, \\ Yangsan-si, Gyeongsangnam-do 50612, Republic of Korea
}

Objectives: HTD treatment is a traditional preventive therapy for neonatal inflammatory diseases such as AD. The aim of this study was to investigate the efficacy of HTD treatments for the maintenance and formation of lipid barrier in Dermatophagoides farina-induced obese NC/Nga mice.

Methods: $20 \mathrm{mg} / \mathrm{kg}$ of CRGR extracts as HTD treatments were orally administered to NC/Nga mice. To induce obesity, high fat diet was served. Dermatophagoides farina extracts was applied on the 4th-6th and 8th-10th weeks to induce AD-like skin lesions in $\mathrm{NC} / \mathrm{Nga}$ mice. Changes of skin conditions in mice were observed by histochemistry and immunohistochemistry.

Results: The results showed that HTD treatments effectively maintained and formed the lipid barrier. In the experimental groups, restorations of Lass2 expression and distributions of filaggrin, involucrin, loricrin, ASM, and LXR means that HTD treatments maintained and generated the lipid barrier. In the dermal papillae, HTD treatments reduced PKC production accompanied by epidermis damage. Furthermore, levels of IL-4, and STAT6 was low. HTD treatment may be effective for preventing inflammation induced by Th2-skewed condition by suppressing the main pathway of Th2 differentiation.

Conclusions: HTD treatment alleviated the inflammatory damage in the skin tissues of the NC/Nga mice by maintaining the lipid barrier and suppressing Th2 differentiation.

Key Words : Hataedock, atopic dermatitis, NC/Nga obese mice, lipid barrier, Th2 differentiation

\section{Introduction}

The prevalence of obesity in children and adolescents continues to increase worldwide. The prevalence of obesity in infants and preschool children in South Korea has increased about 2 times in 10 years ${ }^{1)}$. Recently, several studies have reported that obesity affects the incidence of inflammatory diseases ${ }^{2}$. Furthermore,

\footnotetext{
- Received : 27 November 2018 - Revised : 12 December 2018 Accepted : 12 December 2018

- Correspondence to : Kibong Kim

Department of Korean Pediatrics, Korean Medicine Hospital, Pusan National University, Geumo-ro 20, Mulgeum-eup, Yangsan-si, Gyeongsangnam-do, 50612, Republic of Korea.

Tel : +82-55-360-5952, Fax :+82-55-360-5952, E-mail : kkb@pusan.ac.kr
} 
studies have suggested that obesity induces reduction of the moisturizing and barrier functions of the stratum corneum $(\mathrm{SC})^{3)}$.

Atopic dermatitis (AD) is the most common inflammatory disease of the skin based on the skin barrier dysfunction characterized by pruritus, dryness, and erythematous eczema ${ }^{4)}$. Recently the hypothesis that the pathogenesis of $\mathrm{AD}$ is caused by the destruction of the skin barrier system is emerging ${ }^{5)}$. The defects of skin barrier induced a various abnormalities in lipid composition and the extent of the permeability barrier abnormality in $\mathrm{AD}^{6}$. Thus the abnormalities of the skin barrier are not simply epiphenomenon of $\mathrm{AD}$, but in preference the driver for inflammation of $\mathrm{AD}^{7}$. Damages to skin barrier function have highly interrelation with inflammatory skin disease induction. Recently, Interleukin (IL)-31 that is a cytokine associated with Th2 differentiation in $\mathrm{AD}$, has been shown to reduce the expression of filaggrin, a major constituent of the skin barrier ${ }^{8)}$. In addition, reported that the mutated or reduced filaggrin can cause inflammation by damaging the skin barrier" ${ }^{9}$.

Within traditional Korean medicine, $\mathrm{AD}$ is regarded as related to a fetal heat or fetal poisoning, which are caused by unhealthy dietary habits such as excessive fat intake during pregnancy ${ }^{10)}$. Hataedock (HTD) is traditional treatments of Korean medicine in which herbal extracts are orally administered to neonates to prevent inflammatory diseases caused by mother's dietary habits during pregnancy. Coptidis Rhizoma, Glycyrrhizae Radix (CRGR) are a representative herbal medicine of HTD treatments. Our previous study suggested that HTD treatment maintains and fortifies the lipid barrier ${ }^{11)}$. Based on that study, we sought to study the effect of HTD treatment on the maintenance of lipid barrier in obese conditions that could promote an inflammatory response.

In this study, CRGR was administered orally to 3-week-old NC/Nga mice as the HTD treatments and pediatric obese condition was induced by high fat diet. To investigate the lipid barrier maintenance effect of HTD treatment, Dermatophagoides farinae extract (DfE) was applied to NC/Nga mice to induce AD-like skin lesions. In a series of pathway related to provoking $\mathrm{AD}, \mathrm{HTD}$ treatment is anticipated to assist in maintenance of the lipid barrier. Thus, we investigated the maintenance effects of HTD treatments on the lipid barrier disrupted by DfE in AD-induced obese NC/Nga mice.

\section{Materials and Methods}

\section{Preparation of extracts}

In this study, extracts of Coptidis Rhizoma (Coptis Japonica) and Glycyrrhizae Radix (Glycyrrhiza uralensis) were used for HTD treatment. The CRGR extract was prepared as follows: 1) Coptidis Rhizoma (100 g) and Glycyrrhizae Radix (100 g) were decocted in $1 \mathrm{~L}$ of distilled water for 3 hours and then filtered; 2) The decoction was concentrated to $50 \mathrm{~mL}$ by a rotatory vacuum evaporator, and the filtrate was freeze-dried. As a result, we obtained $31 \mathrm{~g}$ of extract (yield: $15.5 \%$ ).

\section{Experimental animals and induction of $A D$}

Male 3-week-old NC/Nga mice, which were $14.3 \pm 0.3$ $\mathrm{g}$ in weight, were obtained from Central Lab. Animal Inc. (Seoul, Republic of Korea). For diet-induced obesity, diet composed of $60 \%$ fat, $20 \%$ carbohydrates, and $20 \%$ protein was administered. All mice were allowed to eat freely throughout the experiment.

Each 10 mice were allocated to three groups (total 30 mice) as follows: the high fat diet group (Ctrl group), high fat diet and $\mathrm{AD}$-induced group (AE group), and high fat diet and AD-induced group after HTD treatment with CRGR extract group (CGT group). In CGT group, with 3-week-old mice, HTD treatments 
that CRGR extracts $(20 \mathrm{mg} / \mathrm{kg}$ each) are orally administered to each group on the 1st, 2nd, and 3rd days was performed. For AD-like skin lesion, we skinned the backs of mice and swabbed the skinned areas 20 times with $1 \mathrm{~mL}$ of $5 \%$ sodium dodecyl sulfate (SDS; Sigma-Aldrich, St. Louis, MO, USA) using a cotton swab to remove the lipid lamella of the SC. Then, $100 \mathrm{mg}$ of DfE (Biostir Inc., Kobe, Japan) was applied to the mice two times per week. The first exposure was conducted on the 4th, 5th, and 6th weeks. After 1 week, the second exposure was conducted on the 8th, 9th, and 10th weeks. On the 11th week, mice were sacrificed with sodium pentobarbital. The overall procedure is depicted in Fig 1.

All animal experiments were approved by the Institutional Animal Care and Use Committee (IACUC) of Pusan National University (IACUC number: PNU-2015-0924). We also followed the National Institutes of Health (NIH) Guide for the Care and Use of Laboratory Animals throughout this study.

\section{Tissue sample preparation and histochemistry}

After sacrificing the mice, we obtained dorsal skins and then fixed them in $10 \%$ neutral-buffered formalin
(NBF) at room temperature for 24 hours. The fixed tissues were embedded in paraffin for serial sectioning with $5-\mu \mathrm{m}$ thickness. We conducted H\&E (Hematoxylin and Eosin) staining to investigate histological changes including epithelial hyperplasia, capillary distribution, and collagen fiber distribution of the tissue samples.

\section{Immunohistochemistry}

Skin tissue slices were soaked in $20 \mu \mathrm{g} / \mathrm{mL}$ of proteinase $\mathrm{K}$ solution for 5 minutes for proteolysis. The proteolyzed slices were incubated in $10 \%$ normal goat serum for 4 hours, which was used as blocking serum. Next, the slices were treated with primary antibodies (All antibodies used in the experiment were purchased at Santa Cruz Biotechnology, Dallas, TX, USA), including goat anti-Lass2 (1:100), goat anti-filaggrin (1:100), goat anti-involucrin (1:50), goat anti-loricrin (1:50), goat anti- ASM (1:50), goat anti-liver X receptor (LXR; 1:200), goat anti-protein kinase C (1:100), goat anti-IL-4 (1:100), and goat anti-STAT6 (1:100) for 72 hours in a $4{ }^{\circ} \mathrm{C}$ humidified chamber.

Then, the slices reacted with the primary antibodies were treated with biotinylated rabbit anti-goat $\operatorname{IgG}$ (1:100) secondary antibody for 24 hours at room temperature. After reaction with the secondary antibody,
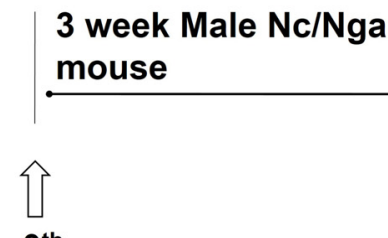

$0^{\text {th }}$

\section{HTD treatment}

\section{HFD feeding}

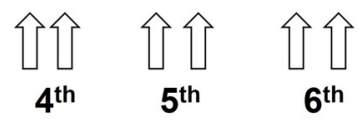

$1^{\text {st }}$ DfE exposure $11^{\text {th }}$ week

Sacrifice

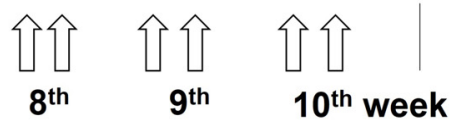

$2^{\text {nd }} D f E$ exposure

Fig. 1. Experiment design.

Prior to induce $A D$, the CRGR extracts for HTD treatment were orally administered to the CGT group on the 1st, 2nd, and 3rd days. Mice (per group $\mathrm{n}=10$ ) were exposed to DfE on the 4th, 5th, and 6th weeks at the first time. On the 8th, 9th, and 10th weeks, the mice were exposed DfE again. Abbreviations: HFD: high fat diet, HTD: Hataedock, and DfE: Dermatophagoides farinae extract. 
the slices were treated with an avidin-biotin complex kit (Vector Laboratories, Burlingame, CA, USA) for 1 hour at room temperature. As a final step, the slices were developed with $0.05 \mathrm{M}$ tris- $\mathrm{HCl}$ buffer solution ( $\mathrm{pH} 7.4$ ) consisting of $0.05 \% 3,3^{\prime}$-diaminobenzidine (DAB) and $0.01 \% \mathrm{HCl}$ and then counterstained with hematoxylin.

\section{Image analysis and statistical analysis}

To obtain numerical data from immunohistochemistry, image analysis was performed by Image Pro Plus (Media cybernetics, Rockville, MD, USA). In the image analysis of 400x-magnified exposure photography, positively reacted particle as pixel cells (intensity range: $80-100$ ) were counted from 10 randomly selected fields from each group. Total pixel cells were 20,000,000 depending on the results of immunohistochemistry such as non-specific structure and artificiality. Data were presented as the mean \pm standard error (mean $\pm \mathrm{SE}$ ). One-way ANOVA and Post-Hoc test were used to analyze statistical significance of the differences with a significance level of $P<0.05$. SPSS 23 software (IBM Corp, Armonk, NY, USA) was used for statistical analysis.

\section{Results}

\section{Alleviating effect on symptoms of $A D$}

$H \& E$ staining was used to observe changes caused by edema including collagen fiber distribution and epithelial hyperplasia. The AE group exhibited pathological change such as reduction in collagen fiber and an increase in epithelial hyperplasia. These results represent the typical histological appearance of inflammatory skin damage induced through persistent application of DfE. In contrast, the CGT group showed less pathological changes than the $\mathrm{AE}$ group in most areas. The CGT group exhibited low epidermal hyperplasia and maintenance of collagen fiber density (Fig 2).

\section{Maintenance of lipid barrier}

To investigate the protective effect of HTD treatment on the epidermal barrier, Lass2-, filaggrin-, involucrin-, and, loricrin- positive reactions were measured.

Lass2-positive reactions were used to detect the ceramide synthesis of the epidermal lipid layer. The Lass2 level of the $\mathrm{AE}$ group was 8,317 $\pm 221 / 20,000,000$ pixel which was decreased in the SC by $90 \%$ compared
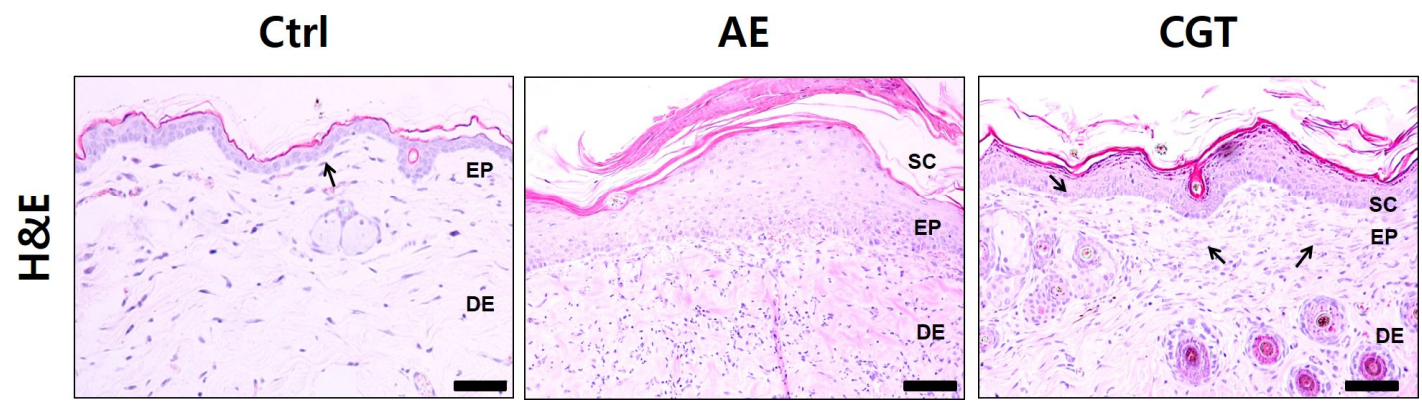

Fig. 2. Alleviating effect on symptoms of $A D$.

HTD treatments treated to the CGT group alleviated the characteristic lesion of AD. In the results of H\&E staining, hyperplasia of the epithelium increased in the AE group but decreased in the CGT group (bar size: $50 \mu \mathrm{m})$. Abbreviations: Ctrl: high fat diet-fed mice, AE: high fat diet-fed and AD-induced mice but no treatment, CGT: high fat diet-fed and AD-induced mice after treated with CRGR extract, EP: epidermis, DE: dermis, and H\&E: Hematoxylin and eosin stain. 
with that of the Ctrl group. Compared with the $\mathrm{AE}$ group, Lass2 levels of the CGT group was increased significantly. The Lass2 level of the CGT group was $34,041 \pm 1320 / 20,000,000$ pixel which was increased by $309 \%(P<0.05)$ compared with that of the AE group (Fig 3).

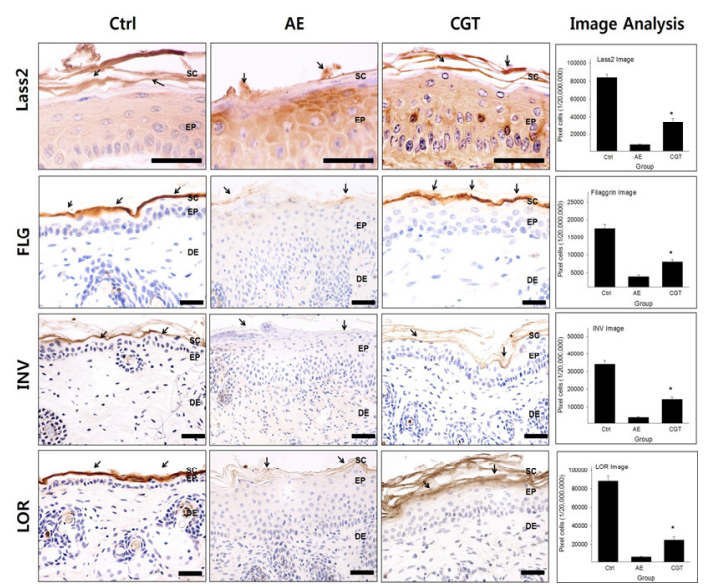

Fig. 3. Maintenance of lipid barrier.

In the results of each immunohistochemistry, both HTD treatments contributed to the maintenance of epidermal barrier. Image analysis data for Lass2-, filaggrin-, involucrin-, loricrin-, ASM-, and LXR-positive reactions (arrows indicating dark brown spot) decreased in the AE group compared with Ctrl group (per group $\mathrm{n}=10)$ (bar size: $50 \mu \mathrm{m})$. In contrast, both HTD treatments showed that Lass2-, filaggrin-, involucrin-, loricrin-, ASM-, and LXR-positive reactions significantly increased in the CGT group (per group $\mathrm{n}=10)$ compared with that of $\mathrm{AE}$ group $(P\langle 0.05)$. Abbreviations: Ctrl: high fat diet-fed mice, $A E$ : high fat diet-fed and AD-induced mice but no treatment, CGT: high fat diet-fed and AD-induced mice after treated with CRGR extract, FLG: filaggrin, INV: involucrin, LOR: loricrin, SC: stratum corneum, EP: epidermis, DE: dermis, and \#: $P<0.05$ compared with the AE group.

Filaggrin-positive reactions were mainly found in keratohyalin granule of SC. The filaggrin level of the AE group was 5,511 $\pm 390 / 20,000,000$ pixel which was decreased by $49 \%$ compared with that of the Ctrl group.
However, both HTD treatments recovered filaggrin levels in the CGT group. The levels of filaggrin in the CGT group was respectively $8,840 \pm 218 / 20,000,000$ pixel which were increased by $60 \%(P<0.05)$ compared with that of the $\mathrm{AE}$ group (Fig 3).

Involucrin-positive reactions were observed in cornified layer of SC. The involucrin level of the AE group was 4,417 $\pm 93 / 20,000,000$ pixel which was decreased by $87 \%$ compared with that of the Ctrl group. In contrast, HTD treatments improved involucrin levels in the CGT group. The levels of involucrin in the CGT group was $14,342 \pm 419 / 20,000,000$ pixel which were significantly increased by $225 \%(P<0.05)$ compared with that of the AE group (Fig 3).

The results for loricrin-positive reaction were similar to the results for involucrin. Compared with the Ctrl group, the loricrin level of the $\mathrm{AE}$ group was $6,501 \pm 227 / 20,000,000$ pixel which was decreased by 93\%. After both HTD treatments, the loricrin levels increased in the CGT group. The loricrin levels of the CGT group was 24,602 $\pm 1090 / 20,000,000$ pixel which was increased by $283 \%(P<0.05)$ compared with that of the AE group (Fig 3).

\section{Formation of lipid barrier}

To investigate the generative effect of HTD treatment on the lipid barrier, ASM- and LXR-positive reactions were measured.

ASM-positive reaction in the stratum granulosum was $3,284 \pm 161 / 20,000,000$ pixel which decreased by 93\% in AE group compared with that of the Ctrl group. However, HTD treatments remarkably increased ASM levels in CGT group. The ASM level of the CGT group was $11,198 \pm 345 / 20,000,000$ pixel which was increased by $240 \%(P<0.05)$ compared with that of the AE group (Fig 4).

LXR-positive reactions were widely exhibited in the cytoplasm of cells in the SC and stratum granulosum. 
Efficacy of Hataedock Treatments for Maintenance and Formation of Lipid Barrier in Obese NCNga Mice with Dermatophagoides Farinae-Induced Atopic Dermatitis

Compared with the Ctrl group, the LXR level was $5,511 \pm 71 / 20,000,000$ pixel which was remarkably decreased by $49 \%$ in the $\mathrm{AE}$ group. In contrast, both HTD treatments significantly improved LXR levels in the CGT group similarly to that of the Ctrl group. The LXR level of the CGT group was 8,840 $\pm 149 / 20,000,000$ pixel which was increased by $60 \%(P<0.05)$ compared with that of the AE group (Fig 4).

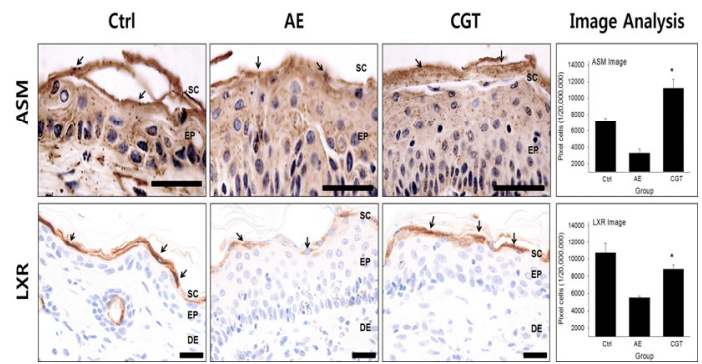

Fig. 4. Formation of lipid barrier.

In the results of each immunohistochemistry, HTD treatments contributed to the formation of lipid barrier. Image analysis data for ASM- and LXR-positive reactions (arrows indicating dark brown spot) decreased in the AE group compared with Ctrl group (per group $n=10$ ) (bar size: 50 $\mu \mathrm{m})$. In contrast, both HTD treatments showed that ASM- and LXR-positive reactions significantly increased in the CGT group (per group $\mathrm{n}=10)$ compared with that of $\mathrm{AE}$ group $(P<0.05)$. Abbreviations: Ctrl: high fat diet-fed mice, AE: high fat diet-fed and $A D$-induced mice but no treatment, CGT: high fat diet-fed and AD-induced mice after treated with CRGR extract, SC: stratum corneum, EP: epidermis, DE: dermis, and \#: $P<0.05$ compared with the AE group.

\section{Regulatory effect on Th2 differentiation}

PKC-, IL-4-, and STAT6-positive reactions were measured to observe the regulatory effect of HTD treatment on $\mathrm{Th} 2$ differentiation in the dermal papilla.

PKC immunohistochemistry was used to detect PKC-positive reactions in damaged epidermis and basement of epidermis. In the AE group, the level of
PKC was $198,283 \pm 3590 / 20,000,000$ pixel which was markedly increased by $5385 \%$ compared with that of the Ctrl group. However, HTD treatments also reduced the levels of PKC in the CGT group by $90 \%(P<0.05)$, compared with that of the AE group. The levels of PKC was 20,016 $\pm 324 / 20,000,000$ pixel in the CGT group (Fig 5).

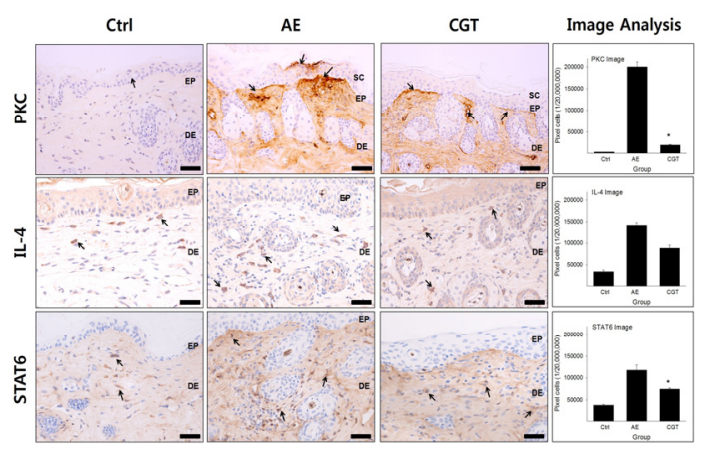

Fig. 5. Regulatory effect on Th2 differentiation. In the immunohistochemistry for PKC-, IL-4-, and STAT6-positive reaction (arrows indicating dark brown spot) decreased both in the CGT group compared with those of the AE group (per group $\mathrm{n}=10$ ) (bar size: $50 \mu \mathrm{m})$. Image analysis data for PKC, IL-4, and STAT6 also showed significant decrease in the CGT group $(P\langle 0.05)$. Abbreviations: Ctrl: high fat diet-fed mice, AE: high fat diet-fed and $A D$-induced mice but no treatment, CGT: high fat diet-fed and $A D$-induced mice after treated with CRGR extract, EP: epidermis, DE: dermis, and \#: $P<0.05$ compared with the $\mathrm{AE}$ group.

The HTD treatments also remarkably reduced the levels of IL-4 and STAT6 compared with the AE group. The IL-4-positive reaction was 556,945 $\pm 5852 / 20,000,000$ pixel which was increased by $977 \%$ compared with that of the Ctrl group. In contrast, the levels of IL-4 in the CGT group was $114,731 \pm 4327 / 20,000,000$ pixel which was decreased by $79 \%(P<0.05)$ compared with that of the AE group (Fig 5). Immunohistochemistry for STAT6 exhibited similar results as those for IL-4. In $\mathrm{AE}$ group, the STAT6-positive reaction was 
$117,672 \pm 3819 / 20,000,000$ pixel which was increased by $210 \%$ compared with that of the Ctrl group. In contrast, levels of STAT6 in the CGT groups was $74,344 \pm 817 / 20,000,000$ pixel which was decreased by $37 \%(P<0.05)$, compared with that of the AE group (Fig 5).

\section{Discussion}

In this study, we investigated the preventive effect of HTD treatment on AD-like skin lesions using DfE - induced obesity NC/Nga mice. H\&E staining was observed to confirm the $\mathrm{AD}$ symptom relief effect of HTD treatments. HTD treatments was observed to alleviate capillary angiogenesis, edema and epithelial hyperplasia. We anticipated that relieved $\mathrm{AD}$ symptoms was due to well-maintained lipid barrier. To observe the effect of HTD treatments on maintaining the lipid barrier, changes of Lass2, filaggrin, involucrin, loricrin, ASM, and LXR levels were measured. HTD treatments helped to maintain the lipid barrier by increasing Lass2, filaggrin, involucrin, loricrin, ASM, and LXR levels. Moreover, we anticipated that HTD treatments relieved $\mathrm{AD}$ symptoms by contributing to the regulation of the Th2 predominant immune response, a major pathological mechanism of $\mathrm{AD}$. To regulate Th2-skewed condition, HTD treatments affected Th2 differentiation by reducing PKC, IL-4, and STAT6 levels.

The hypothesis for etiopathogenesis of $\mathrm{AD}$ has shifted from "inside to outside" to "outside to inside" The "inside to outside" hypothesis suggests that the epidermal barrier is secondarily damaged due to rising inflammation caused by the imbalance between Th1 and Th2 cytokines, which is known as "Th2-skewed condition" $"$. However, the recent "outside to inside" hypothesis focuses on filaggrin. Filaggrin plays an important role in maintaining the SC by modulating hydration and $\mathrm{pH}$ level ${ }^{14)}$. The "outside to inside" hypothesis proposes that the defective SC due to impaired filaggrin stimulates the Th2 response by promoting persistent secretion of pro-inflammatory cytokines $^{13,14)}$.

As mentioned earlier, obesity and inflammatory response are correlated. In particular, adipocytes produce and secrete cytokines such as TNF- $\alpha$, IL-6, and monocyte chemotactic activating factor (MCP) -1 , which play a central role in the inflammatory response ${ }^{15}$. Actually in obese subjects, an increase in pro-inflammatory cytokines such as TNF-alpha and IL-6 in the blood and an increase in CRP (c-reactive protein) as a biomarker of inflammatory diseases are observed ${ }^{16)}$. Furthermore, many macrophages accumulate in adipocytes, leading to inflammation of the obese tissue itself, leading to chronic inflammation ${ }^{17)}$.

Thus, we tried to confirm the efficacy of treatment of HTD treatments on AD by observing the levels of protein constituting lipid barrier in the obese conditions promoting the inflammatory response.

\section{Alleviating effect on symptoms of $A D$}

$\mathrm{AD}$ is characterized by pruritic skin lesions, impaired epidermal lipid barrier function, imbalance of the immune system, and allergic reactions ${ }^{18)}$. Macroscopically, the HTD treatments alleviated AD symptoms such as edema including collagen fiber distribution and epithelial hyperplasia in obese $\mathrm{NC} / \mathrm{Nga}$ mice. Furthermore, HTD treatment reduced angiogenesis. Angiogenesis of capillaries promoted by VEGFs is a hallmark of chronic inflammatory diseases such as AD. Angiogenesis is closely related to inflammation response by molecular links that cells involved in the inflammatory process release factors acting on the vascular endothelial cells ${ }^{19)}$. Moreover, angiogenesis maintains inflammation by supplying oxygen and nutrients to cells in the inflammatory area ${ }^{20)}$. 


\section{Maintenance of lipid barrier}

Lass2, filaggrin, involucrin, and loricrin are closely related to maintenance of the epidermal lipid barrier. Ceramide is a typical substance to keep moisture and to form permeability barrier function in SC. Reduced ceramides in SC can cause skin abnormalities such as $\mathrm{AD}$ due to loss of protection against antigens, moisture loss from the epidermis, and dysfunction of barrier ${ }^{21}$. Among the Lass recently named ceramide synthase (CerS), Lass2 that catalyzes the synthesis of very long acyl chain ceramides is the most commonly expressed of all CerS and is most widely distributed in the human body ${ }^{22)}$. Deficiency of ceramide synthesis containing heavy chain fatty acids by Lass 2 causes severe skin diseases $^{23)}$. A reduced level of ceramide and changes in ceramide subclass were observed in $\mathrm{AD}$ or psoriasis ${ }^{24}$, and the replacement of ceramide to short chain lengths was reported in patients with $\mathrm{AD}^{25)}$.

Filaggrin mutations causes skin barrier damage in $\mathrm{AD}$ and promotes IgE sensitization due to damaged skin barrier ${ }^{26)}$. Although there are many causes for filaggrin reduction, particularly Th2-skewed condition contributes to reducing filaggrin levels in $\mathrm{AD}$ patient ${ }^{14)}$. $\mathrm{AD}$ symptoms was relieved by improving the skin barrier due to increased expression of filaggrin ${ }^{27)}$. Involucrin and loricrin are down-regulated by IL-4 in keratinocytes that process plays an important role in AD pathogenesis ${ }^{28)}$. Particularly, decreased expression of involucrin and loricrin is inhibited in knock-out STAT6 mice, suggesting that down-regulation by IL-4 is mediated through a STAT6-dependent mechanism ${ }^{29}$.

In our previous study, we suggested that HTD treatment significantly increased filaggrin, involucrin, loricrin, and ASM levels, which are critical to the differentiation of epithelial cells and epidermal barrier function $^{30)}$. Consistent with previous findings, HTD treatments used in this study increased the level of Lass2, filaggrin, involucrin, and loricrin. Based on this result, HTD treatments may help to fortify the epidermal lipid barrier.

\section{Formation of lipid barrier}

ASM and LXR are closely related to formation of the epidermal lipid barrier. ASM, an enzyme that converts phospholipids into ceramides, also plays an important role in the formation of ceramides for epidermal barrier function ${ }^{31)}$. ASM activity has been reported to be significantly reduced in $\mathrm{AD}$ lesions ${ }^{32}$, and reduced ASM activity is thought to be responsible for the reduced ceramide level, which is specific found in $\mathrm{AD}^{33}$. In addition, deficiency of ASM in $\mathrm{AD}$ inhibits recovery after damage to the skin barrier ${ }^{32)}$ and the reduction of ASM activity in $\mathrm{AD}$ leads to skin barrier disorders by decreasing ceramide, involucrin, loricrin, and filaggrin ${ }^{34)}$. In skin, activation of LXR improves permeability barrier homeostasis by stimulating epidermal lipid synthesis ${ }^{35)}$, and promotes recovery of permeability barrier damage ${ }^{36}$. In addition, LXR controls cutaneous inflammation by inhibiting secretion of cytokine such as TNF- $\alpha$ and IL-1a that mediate the cutaneous inflammatory response ${ }^{37)}$.

In this study, we compared the levels of ASM and LXR in order to examine the generative effect of HTD treatment on epidermal lipid barrier. The result showed that HTD treatment affected production of the levels of ASM and LXR. Based on this result, HTD treatments may help to generate the epidermal lipid barrier.

\section{Regulatory effect on Th2 differentiation}

Infiltrated external allergens can exacerbate Th2-skewed condition by activating inflammatory cells, including Th2 cells. For Th2 differentiation during inflammation, STAT6 signaling is an important pathway, and IL-4 is upstream of STAT6 ${ }^{38)}$. STAT6 is closely related to IL-4 and IL-13 signaling, regulates important gene expression to IL-4 function and Th2 
differentiation ${ }^{39), 40)}$. In a knock-out STAT6 mice model, Th2 response by IL-4 does not occur ${ }^{41)}$, and showed a loss of Th2 cell accumulation, which is one of the main features of asthma ${ }^{42)}$. IL-4 is one of the key cytokines for Th2 differentiation and subsequent inflammation since it affects immune cells such as $\mathrm{T}$ cell, B cell, and macrophage through the $\alpha$ subunit of IL-4 receptor (IL-4R $\alpha)^{38,43)}$. CD4 cells of knock-out IL-4 mice were unable to produce Th2 cytokines ${ }^{44)}$. In additions, PKC is absolutely essential to differentiation of naive $\mathrm{T}$ cells into Th2 cells ${ }^{45)}$ and PKC regulates the IL-4/STAT6 signaling pathway in $\mathrm{T}$ cells by phosphorylating Janus kinase (Jak) $1^{46}$. PKC plays an important role in the Jak1/STAT6 signaling cascade associated with activation through IL-4 and Th2 differentiation. Moreover, PKC activation is important for epidermal inflammation since keratinocytes secreting inflammatory mediators such as TNF- $\alpha$ and cyclooxygenase-2 (COX-2) require PKC activation for appropriate differentiation ${ }^{47}$.

Based on these results, we compared the levels of IL-4, STAT-6 and PKC in order to examine the regulatory effect of HTD treatment on Th2 differentiation. In this study, HTD treatment affected reduction of the levels of IL-4, STAT6, and PKC. Down-regulation of IL-4 and PKC were also observed in our previous study ${ }^{48)}$, but STAT6-inhibitory activity was reported here for the first time. Considering our results, HTD treatment may be effective for preventing inflammation induced by Th2-skewed condition by suppressing the main pathway of Th2 differentiation.

In previous studies, we demonstrated that HTD treatments reduce the inflammatory response by down-regulating Th2 differentiation ${ }^{48)}$. In addition, we demonstrated that HTD treatments maintains and fortifies the lipid barrier ${ }^{11}$. Based on these results, we hypothesize that HTD could attenuate AD symptoms by maintaining lipid barrier in obese conditions, an aggravating factor of inflammation. In conclusion, the HTD treatments contributed to down-regulation of Th2 differentiation in AD-induced obese $\mathrm{NC} / \mathrm{Nga}$ mice, leading to the alleviation of $\mathrm{AD}$ symptoms by maintaining the lipid barrier. Considering that the prevalence of childhood obesity is increasing, these results suggest that HTD treatments may contribute as an alternative treatment for the prevention of inflammatory diseases.

\section{Conclusions}

Obesity can aggravate inflammatory diseases. However, in this study, CRGR HTD treatment alleviated the inflammatory damage in the skin tissues of the $\mathrm{NC} / \mathrm{Nga}$ mice by maintaining the lipid barrier and suppressing Th2 differentiation.

\section{Acknowledgements}

This study was supported by the Basic Science Research program through the National Research Foundation of Korea (NRF) funded by the Ministry of Education [No. NRF-2016R1D1A1B03930474].

\section{References}

1. Kang KS. Nutritional Counseling for Obese Children with Obesity-Related Metabolic Abnormalities in Korea. Pediatr Gastroenterol Hepatol Nutr. 2017;20(2):71-8.

2. Silverberg JI, Kleiman E, Lev-Tov H, Silverberg NB, Durkin HG, Joks R, et al. Association between obesity and atopic dermatitis in childhood: A case-control study. J Allergy Clin Immunol. 2011;127(5):1180-6.

3. Mori S, Shiraishi A, Epplen K, Butcher D, Murase D, Yasuda $Y$, et al. Characterization of skin 
function associated with obesity and specific correlation to local/systemic parameters in American women. Lipids Health Dis. 2017; 16(1):214.

4. Peng W, Novak N. Pathogenesis of atopic dermatitis. Clin Exp Allergy. 2015;45(3):566-74.

5. Kubo A, Nagao K, Amagai M. Epidermal barrier dysfunction and cutaneous sensitization in atopic diseases. J Clin Invest. 2012;122(2):440-7.

6. Elias PM. Lipid abnormalities and lipid-based repair strategies in atopic dermatitis. Biochim Biophys Acta. 2014;1841(3):323-30.

7. Taieb A. Hypothesis: from epidermal barrier dysfunction to atopic disorders. Contact Dermatitis. 1999;41(4):177-80.

8. Cornelissen C, Marquardt Y, Czaja K, Wenzel J, Frank J, Luscher-Firzlaff J, et al. IL-31 regulates differentiation and filaggrin expression in human organotypic skin models. J Allergy Clin Immunol. 2012;129(2):426-33.

9. Kim HJ, Shin JU, Lee KH. Atopic dermatitis and skin barrier dysfunction. Allergy Asthma Respir Dis. 2013;1(1):20-8.

10. Romieu I, Torrent M, Garcia-Esteban R, Ferrer C, Ribas-Fito N, Anto JM, et al. Maternal fish intake during pregnancy and atopy and asthma in infancy. Clin Exp Allergy. 2007;37(4):518-25.

11. Kim HY, Ahn SH, Yang IJ, Kim K. Effect of Skin Lipid Barrier Formation on Hataedock Treatment with Douchi. J Korean Med. 2017; 38(2):41-52.

12. Jurakic Toncic R, Marinovic B. The Role of Impaired Epidermal Barrier Function in Atopic Dermatitis. Acta Dermatovenerol Croat. 2016;24(2):95-109.

13. Elias PM, Hatano Y, Williams ML. Basis for the barrier abnormality in atopic dermatitis: outsideinside-outside pathogenic mechanisms. J Allergy Clin Immunol. 2008;121(6):1337-43.
14. Thyssen JP, Kezic S. Causes of epidermal filaggrin reduction and their role in the pathogenesis of atopic dermatitis. J Allergy Clin Immunol. 2014;134(4):792-9.

15. Trayhurn P, Wood IS. Adipokines: inflammation and the pleiotropic role of white adipose tissue. Br J Nutr. 2004;92(3):347-55.

16. Bistrian BR, Khaodhiar L. Chronic systemic inflammation in overweight and obese adults. JAMA. 2000;283(17):2235.

17. Weisberg SP, McCann D. Desai M, Rosenbaum M, Leibel RL, Ferrante AW Jr.. Obesity is associated with macrophage accumulation in adipose tissue. J Clin Invest. 2003;112(12): 1796-808.

18. Kanchongkittiphon W, Gaffin JM, Phipatanakul W. Child with atopic dermatitis. Ann Allergy Asthma Immunol. 2015;114(1):6-11.

19. Benelli R, Lorusso G, Albini A, Noonan DM. Cytokines and chemokines as regulators of angiogenesis in health and disease. Curr Pharm Des. 2006;12(24):3101-15.

20. Charo IF, Taubman MB. Chemokines in the pathogenesis of vascular disease. Circ Res. 2004;95(9):858-66.

21. Holleran WM, Takagi Y, Uchida Y. Epidermal sphingolipids: metabolism, function, and roles in skin disorders. FEBS Lett. 2006;580(23):5456-66.

22. Laviad EL, Albee L, Pankova-Kholmyansky I, Epstein S, Park H, Merrill AH, Jr., et al. Characterization of ceramide synthase 2: tissue distribution, substrate specificity, and inhibition by sphingosine 1-phosphate. J Biol Chem. 2008;283(9):5677-84.

23. Li W, Sandhoff R, Kono M, Zerfas P, Hoffmann V, Ding BC, et al. Depletion of ceramides with very long chain fatty acids causes defective skin permeability barrier function, and neonatal lethality in ELOVL4 deficient mice. Int J Biol 
Sci. 2007;3(2):120-8.

24. Imokawa G, Abe A, Jin K, Higaki Y, Kawashima M, Hidano A. Decreased level of ceramides in stratum corneum of atopic dermatitis: an etiologic factor in atopic dry skin? J Invest Dermatol. 1991;96(4):523-6.

25. Janssens M, van Smeden J, Gooris GS, Bras W, Portale G, Caspers PJ, et al. Increase in short-chain ceramides correlates with an altered lipid organization and decreased barrier function in atopic eczema patients. J Lipid Res. 2012;53(12): 2755-66.

26. O'Regan GM, Irvine $\mathrm{AD}$. The role of filaggrin in the atopic diathesis. Clin Exp Allergy. 2010;40(7):965-72.

27. Kim H, Kim JR, Kang H, Choi J, Yang H, Lee $\mathrm{P}$, et al. 7,8,4'-Trihydroxyisoflavone Attenuates DNCB-Induced Atopic Dermatitis-Like Symptoms in NC/Nga Mice. PLoS One. 2014;9(8): e104938. 28.

28. Bao L, Mohan GC, Alexander JB, Doo C, Shen $\mathrm{K}$, Bao J, et al. A molecular mechanism for IL-4 suppression of loricrin transcription in epidermal keratinocytes: implication for atopic dermatitis pathogenesis. Innate Immun. 2017;23(8):641-7.

29. Bao L, Alexander JB, Zhang H, Shen K, Chan LS. Interleukin-4 Downregulation of Involucrin Expression in Human Epidermal Keratinocytes Involves Stat6 Sequestration of the Coactivator CREB-Binding Protein. J Interferon Cytokine Res. 2016;36(6):374-81.

30. Steinert PM, Marekov LN. The proteins elafin, filaggrin, keratin intermediate filaments, loricrin, and small proline-rich proteins 1 and 2 are isodipeptide cross-linked components of the human epidermal cornified cell envelope. J Biol Chem. 1995;270(30):17702-11.

31. Schmuth M, Man MQ, Weber F, Gao W, Feingold KR, Fritsch P, et al. Permeability barrier disorder in Niemann-Pick disease: sphingomyelin-ceramide processing required for normal barrier homeostasis. J Invest Dermatol. 2000;115(3):459-66.

32. Pullmannova P, Stankova K, Pospisilova M, Skolova B, Zbytovska J, Vavrova K. Effects of sphingomyelin/ceramide ratio on the permeability and microstructure of model stratum corneum lipid membranes. Biochim Biophys Acta. 2014;1838(8): 2115-26.

33. Di Nardo A, Wertz P, Giannetti A, Seidenari S. Ceramide and cholesterol composition of the skin of patients with atopic dermatitis. Acta Derm Venereol. 1998;78(1):27-30.

34. Jensen JM, Folster-Holst R, Baranowsky A, Schunck M, Winoto-Morbach S, Neumann C, et al. Impaired sphingomyelinase activity and epidermal differentiation in atopic dermatitis. $\mathrm{J}$ Invest Dermatol. 2004;122(6):1423-31.

35. Man MQ, Choi EH, Schmuth M, Crumrine D, Uchida Y, Elias PM, et al. Basis for Improved Permeability Barrier Homeostasis Induced by PPAR and LXR Activators: Liposensors Stimulate Lipid Synthesis, Lamellar Body Secretion, and Post-Secretory Lipid Processing. J Invest Dermatol. 2006;126(2):386-92.

36. Delvecchio CJ, Capone JP. Protein kinase C alpha modulates liver $\mathrm{X}$ receptor alpha transactivation. J Endocrinol. 2008;197(1):121-30.

37. Schmuth M, Jiang YJ, Dubrac S, Elias PM, Feingold KR. Thematic review series: skin lipids. Peroxisome proliferator-activated receptors and liver $\mathrm{X}$ receptors in epidermal biology. J Lipid Res. 2008;49(3):499-509.

38. Walford HH, Doherty TA. STAT6 and lung inflammation. JAKSTAT. 2013;2(4):e25301.

39. Hershey GK. IL-13 receptors and signaling pathways: an evolving web. J Allergy Clin Immunol. 2003;111(4):677-90.

40. Hebenstreit D, Wirnsberger G, Horejs-Hoeck J, 
Duschl A. Signaling mechanisms, interaction partners, and target genes of STAT6. Cytokine Growth Factor Rev. 2006;17(3):173-88.

41. Shimoda K, van Deursen J, Sangster MY, Sarawar SR, Carson RT, Tripp RA, et al. Lack of IL-4-induced Th2 response and IgE class switching in mice with disrupted Stat6 gene. Nature. 1996;380(6575):630-3.

42. Mathew A, MacLean JA, DeHaan E, Tager AM, Green FH, Luster AD. Signal transducer and activator of transcription 6 controls chemokine production and $\mathrm{T}$ helper cell type 2 cell trafficking in allergic pulmonary inflammation. J Exp Med. 2001;193(9):1087-96.

43. Matsunaga MC, Yamauchi PS. IL-4 and IL-13 Inhibition in Atopic Dermatitis. J Drugs Dermatol. 2016;15(8):925-9.

44. Kopf M, Le Gros G, Bachmann M, Lamers MC, Bluethmann H, Kohler G. Disruption of the murine IL-4 gene blocks Th2 cytokine responses. Nature. 1993;362(6417):245-8.

45. Martin P, Villares R, Rodriguez-Mascarenhas S, Zaballos A, Leitges M, Kovac J, et al. Control of $\mathrm{T}$ helper 2 cell function and allergic airway inflammation by PKCzeta. Proc Natl Acad Sci U S A. 2005;102(28):9866-71.

46. Duran A, Rodriguez A, Martin P, Serrano M, Flores JM, Leitges M, et al. Crosstalk between PKCzeta and the IL4/Stat6 pathway during T-cell-mediated hepatitis. EMBO J. 2004;23(23):4595-605.

47. Denning MF. Epidermal keratinocytes: regulation of multiple cell phenotypes by multiple protein kinase $\mathrm{C}$ isoforms. Int $\mathrm{J}$ Biochem Cell Biol. 2004;36(7):1141-6.

48. Jung AR, Ahn SH, Park IS, Park SY, Jeong SI, Cheon JH, et al. Douchi (fermented Glycine max Merr.) alleviates atopic dermatitis-like skin lesions in NC/Nga mice by regulation of PKC and IL-4. BMC Complement Altern Med. 2016;16(1):416.

\section{ORCID}

Hee-Yeon Kim: https://orcid.org/0000-0002-9693-1840

Sang-Hyun Ahn: https://orcid.org/0000-0002-1526-4397

In-Jun Yang: https://orcid.org/0000-0002-6113-1325

Jin-Hong Cheon: https://orcid.org/0000-0002-6472-1128

Kibong Kim: 